\title{
Principios en técnicas de suturas de piel: una guía para estudiantes
}

\author{
Adriana Marcela González-Cely* \\ Audrey Miranda-Díaz** \\ Juan Dario Alviar***
}

\begin{abstract}
"Médico. Residente IV año posgrado de cirugía plástica. Universidad Industrial de Santander. Bucaramanga. Santander. Colombia.
"Médico. Residente III año posgrado de cirugía plástica. Universidad Industrial de Santander. Bucaramanga. Santander. Colombia.

*** Cirujano plástico. Universidad Nacional de Colombia. Profesor posgrado de cirugía plástica. Universidad Industrial de Santander. Bucaramanga.

Correspondencia: Dr. Audrey Miranda Diaz. Dirección: Calle 20 \#31-27 Apto 703, Condominio Viaggio. Bucaramanga. Santander. Colombia. Correo electrónico: amdrey321@gmail.com
\end{abstract}

Resumen

Introducción: las heridas en la piel son un evento altamente frecuente en los servicios de urgencias, las cuales en su mayoría van a requerir ser suturadas por el personal de salud disponible, tratando de lograr buenos resultados funcionales y cosméticos. Este procedimiento puede definirse como la aproximación de los bordes de una herida por medio de puntos, utilizando suturas, instrumental quirúrgico y agujas adecuadas dependiendo del área lesionada; para lograr una adecuada sutura se requieren conceptos claros como tipo de sutura y aguja, técnica quirúrgica, localización anatómica de la herida y cierre sin tensión. Objetivo: guiar la adecuada práctica de técnicas de suturas de piel por parte de estudiantes y profesionales en el área de la salud. Temática: en este artículo se realizó una descripción precisa de cada uno de los pasos para lograr una adecuada sutura de piel, priorizando en el uso de ilustraciones para alcanzar un mejor aprendizaje. Conclusiones: los resultados en la realización de una sutura de piel dependerán de un adecuado conocimiento y uso del material e instrumental quirúrgico además del dominio de los principios básicos de la técnica quirúrgica. MÉD.UIS. 2018;31(2):65-76.

Palabras clave: Suturas. Técnicas de sutura. Piel. Cuidados de la Piel. Procedimientos Quirúrgicos Reconstructivos.

\section{Principles of skin suture techniques: a student guide}

\section{Abstract}

Introduction: skin wounds are a highly frequent event presenting in the emergency department, most of which require to be sutured by the health care givers available at the moment, while trying to obtain good functional and aesthetic results. This procedure can be defined as the approximation of wound edges by stitches requiring special surgical instruments and sutures depending on the affected area; to accomplish an adequate skin suture clear concepts of the different types of materials, surgical technique, anatomic location and free tension closure are needed. Objective: to provide an adequate guide in suture techniques to medical students and health care professionals. Methods: a precise description of the different steps to obtain an adequate skin suture is made in this article, giving great importance to the use of illustrations to facilitate a better learning process. Conclusions: the results obtained after the making of a skin suture will depend on adequate knowledge, the use of appropriate materials and instruments and the complete grasp of the basic principles in surgical technique. MÉD.UIS. 2018;31(2):65-76.

Keywords: Suture. Suture Techniques. Skin. Skin Care. Reconstructive Surgical Procedures.

¿Cómo citar este artículo?: González-Cely A, Miranda-Díaz A, Alviar J. Principios en técnicas de suturas de piel: una guía para estudiantes. MÉD.UIS. 2018;31(2):65-76. Páginas. DOI: 10.18273/ revmed.v31n2-2018008

Artículo recibido el 4 de enero de 2018 y aceptado para publicación el 19 de julio de 2018 


\section{Introducción}

Las heridas en la piel son un evento altamente frecuente en los servicios de urgencias, las cuales en su mayoría van a requerir ser suturadas por el personal de salud disponible, (en algunos casos con poco entrenamiento en suturas) quien no debe conformarse solamente con realizar el cierre de la piel, sino también con lograr los mejores resultados funcionales y cosméticos posibles. Este procedimiento puede definirse como la aproximación de los bordes de una herida por medio de puntos, utilizando suturas, instrumental quirúrgico y agujas adecuadas dependiendo del área lesionada. De forma contraria, el desconocimiento del instrumental quirúrgico y su inadecuado uso puede llevar a resultados insatisfactorios y/o mayor tiempo de realización del procedimiento ${ }^{1,2}$.

Para la realización de una sutura con hilo óptima se deben tener en cuenta diversas consideraciones técnicas como el calibre de la sutura y tamaño de la aguja, cuya elección dependerá de la región anatómica y el grosor de la piel; además, el manejo atraumático de los tejidos, las diferentes capas anatómicas para suturar dependiendo del sitio, la profundidad y la complejidad de la herida son igualmente puntos críticos para lograr una adecuada sutura $^{1-3}$. Estas consideraciones tienen como objetivo obtener los mejores resultados y adicionalmente disminuir el riesgo de complicaciones como dehiscencia o infección.

Este artículo tiene como objetivo guiar la adecuada práctica de técnicas de suturas de piel por parte de estudiantes y profesionales en el área de la salud teniendo en cuenta el instrumental quirúrgico adecuado, uso de anestésicos locales, los principios básicos de suturas, selección del tipo de punto según el tipo de herida y cada uno de los paso para obtener un resultado satisfactorio ya sea funcional o cosmético además de recomendaciones posterior al procedimiento $^{1,2}$.

\section{Materiales y métodos}

Se realizó una búsqueda de la literatura en las bases de datos PubMed, Embase, Scielo, Lilacs y Science direct; utilizando las palabras clave: Suturas; Técnicas de sutura; Piel; Cuidados de la Piel; Procedimientos Quirúrgicos Reconstructivos. Encontrándose 15 artículos, de los cuales 8 fueron relevantes para esta revisión de tema sobre principios en técnicas de suturas de piel, complementados con 12 capítulos de libros. Se realizó una revisión de los mismos y sobre estos un resumen de los principios en técnicas de suturas de piel, desde la descripción del instrumental que se utiliza, los anestésicos locales disponibles, tipos de suturas, principios para un cierre óptimo de heridas, teniendo en cuenta los diferentes pasos para suturar una herida, con una explicación gráfica de los puntos de sutura descritos en la literatura, que permiten obtener un cierre óptimo de lesiones en la piel para finalmente puntualizar en los cuidados y recomendaciones a tener en cuenta luego de realizar este procedimiento.

\section{Instrumental para sutura de piel}

El instrumental básico necesario para realizar una sutura en piel consta de cuatro elementos: porta agujas, pinza de disección, tijeras y bisturí (Ver Figura 1), cada uno de estos instrumentos tiene funciones específicas y una forma adecuada para sujetarlos con lo que se logra una mejor manipulación de los tejidos y consecuentemente una sutura de mayor calidad $^{1,2}$.

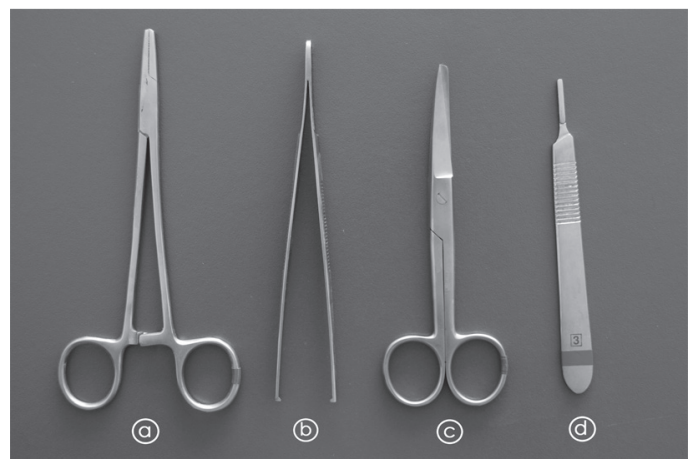

Figura 1. Instrumental básico para sutura de piel. a) Porta agujas. b) Pinza de disección. c) Tijeras. d) Bisturí.

Fuente: autores

\section{Porta agujas}

Es un instrumento diseñado para tomar las agujas con precisión y seguridad, conduciéndola a través de los tejidos. El porta agujas debe sostener la aguja por el cuerpo (en la mitad) y no en los extremos; además, puede sujetarse de dos formas: introduciendo o sin introducir los dedos dentro de sus orificios; se logran movimientos de mayor prono-supinación de la muñeca sin introducir los dedos dentro de los orificios $^{1,2}$ (Ver Figura 2). 
Mayo - agosto
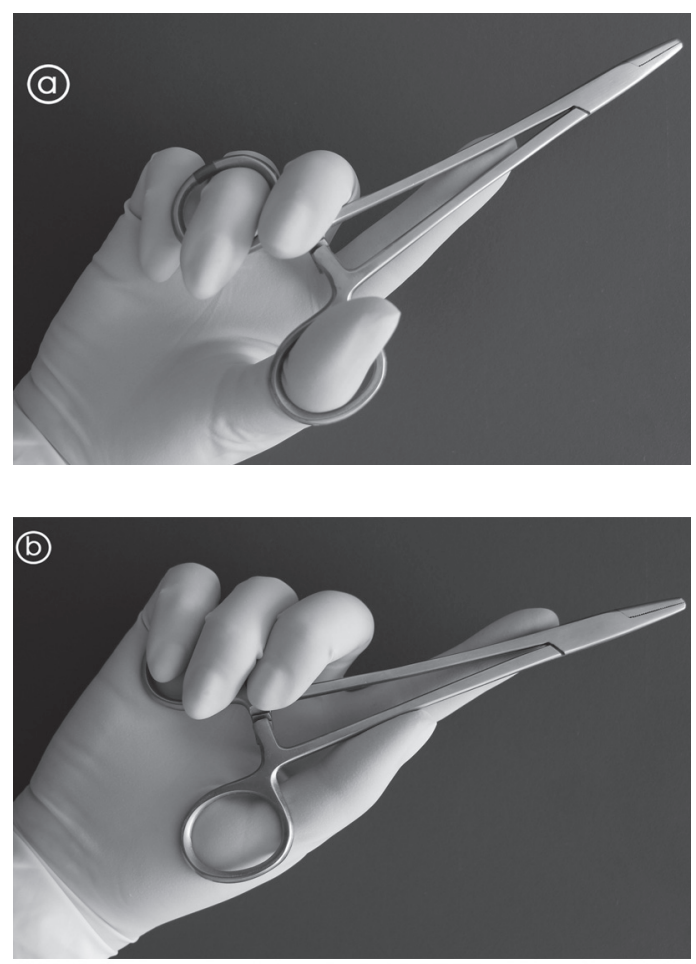

Figura 2. Formas de sujetar el porta agujas. a) Introducir los dedos anular y pulgar en los orificios del instrumental, el dedo índice en el domo como apoyo. b) Orificios sujetados con los tres últimos dedos de la mano y con la región tenar, dedo índice en el domo del instrumento.

Fuente: autores

\section{Pinza de disección}

Empleado para sujetar y traccionar los bordes de la herida y evertirlos durante el paso del punto. Están disponibles pinzas de disección con garra y sin garra. En la piel se deben usar pinzas con garra, ya que causan menor trauma al tener una menor superficie de contacto. La forma correcta de sujetarlas es como se tomaría un lápiz ${ }^{1,2}$ (Ver Figura 3).

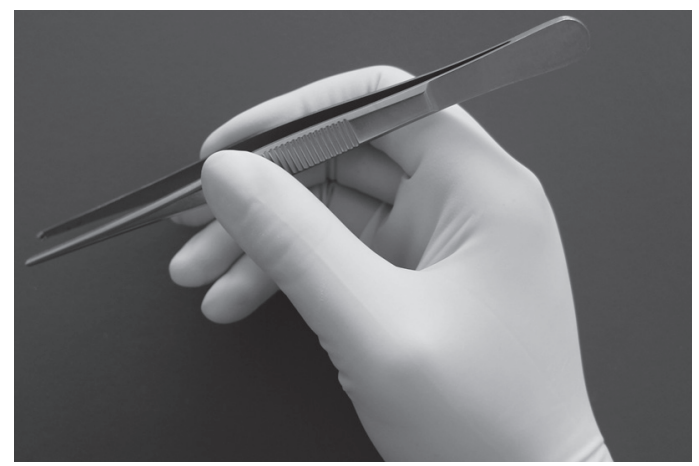

Figura 3. Pinzas de disección. La forma correcta de sujetarlas es como se tomaría un lápiz.

Fuente: autores.
Principios en técnicas de suturas de piel: una guía para estudiantes

\section{Tijeras}

Las tijeras de Mayo se usan para cortar el material de sutura, gasas o vendajes. Además, para regularizar los bordes de la herida en caso de ser necesario o resecar tejido desvitalizado. Se deben tomar de la misma forma que el porta agujas ${ }^{1,2}$ (Ver Figura 4).

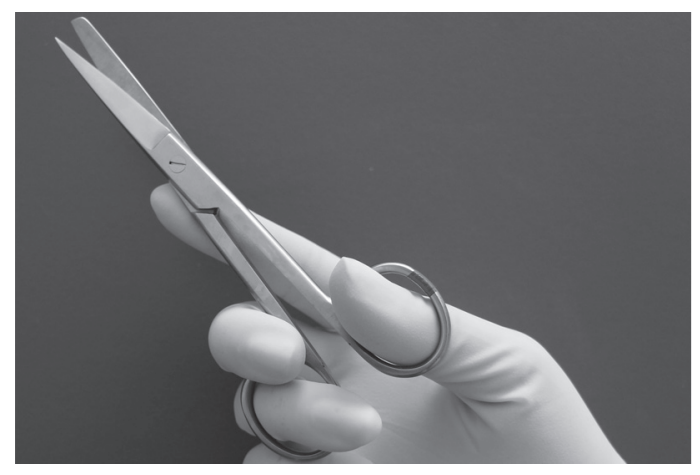

Figura 4. Tijeras de material. Forma correcta de sujetarlas como si se tomara un porta agujas.

Fuente: autores

\section{Bisturí}

Consta de un mango y una hoja de bisturí, lo que permite un corte preciso sobre la piel y otros tejidos; se toma en la mano como sujetando un lápiz y para incidir la piel debe estar de manera perpendicular a esta", 2 (Ver Figura 5).

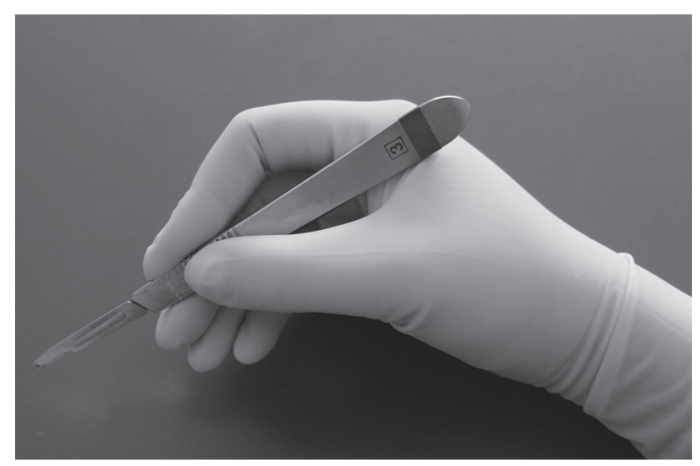

Figura 5. Bisturí. Forma correcta de sujetarlo, como sujetando un lápiz.

Fuente: autores

\section{Anestésicos locales}

El uso de anestésicos locales es indispensable para realizar de forma cómoda y segura las suturas de piel; adicionalmente van a permitir una mejor valoración, lavado y cierre de la misma. Los anestésicos locales disponibles son tipo amida, como lidocaína al 0.5\%, 
$1 \%$ o $2 \%$ y bupivacaina al $0.25 \%$ cuyas dosis a utilizar dependerán del peso de la persona ${ }^{3-5}$ (Ver Tabla 1). Se prefiere el uso de anestésicos locales con epinefrina, dado que aumenta la amplitud de la duración del efecto anestésico, disminuye el sangrado y permite utilizar dosis mayores del mismo.

A pesar del mito que prohíbe usar epinefrina en regiones anatómicas terminales como puntas de dedos y la nariz, la evidencia científica muestra que es seguro usar epinefrina en estas áreas en dosis adecuadas $^{6,7}$.

Tabla 1. Anestésicos locales más utilizados

\begin{tabular}{|c|c|c|c|}
\hline Anestésico & $\begin{array}{c}\text { Dosis } \\
\text { máxima }\end{array}$ & $\begin{array}{l}\text { Comienzo de } \\
\text { acción }\end{array}$ & Duración \\
\hline $\begin{array}{l}\text { Lidocaína 1\% (10 } \\
\text { mg/ml) }\end{array}$ & $\begin{array}{l}4 \mathrm{mg} / \mathrm{kg} \text { de } \\
\text { peso }\end{array}$ & \multirow{2}{*}{ Rápido (3 min) } & $30-120 \mathrm{~min}$ \\
\hline $\begin{array}{l}\text { Lidocaína 1\% con } \\
\text { epinefrina (10 mg/ } \\
\text { ml) }\end{array}$ & $\begin{array}{l}7 \mathrm{mg} / \mathrm{Kg} \text { de } \\
\text { peso }\end{array}$ & & $60-180 \mathrm{~min}$ \\
\hline $\begin{array}{l}\text { Bupivacaína } \\
(0.25 \%)\end{array}$ & $\begin{array}{l}1.5-2.5 \\
\mathrm{mg} / \mathrm{Kg}\end{array}$ & \multirow{2}{*}{ Lento (15 min) } & $\begin{array}{l}120-240 \\
\min \end{array}$ \\
\hline $\begin{array}{l}\text { Bupivacaina con } \\
\text { epinefrina }(0.25 \%)\end{array}$ & $3 \mathrm{mg} / \mathrm{Kg}$ & & $240-480 \mathrm{~min}$ \\
\hline
\end{tabular}

Fuente: autores

\section{Materiales de sutura}

Existen diferentes tipos de materiales de sutura como hilos, grapas, suturas adhesivas y adhesivos tisulares. Los hilos de sutura varían en su origen (natural o sintético), tiempo de permanencia en el tejido, configuración, elasticidad, memoria, capilaridad, visibilidad, y costo. Pueden clasificarse de acuerdo a su permanencia en los tejidos como absorbibles y no absorbibles ${ }^{2,8}$ (Ver Tabla 2). Para suturar la piel, se utilizan suturas no absorbibles las cuales generan menos reacción en los tejidos y se deben retirar en determinado tiempo de acuerdo a la región corporal donde se encuentren. Las suturas absorbibles se utilizan para puntos en mucosa, puntos de afrontamiento subdérmicos o en tejido celular subcutáneo cuando las heridas son muy profundas y se busca una mejor aproximación de los tejidos, disminución de tensión en los bordes de la piel y cierre de un tercer espacio; estas suturas absorbibles también se pueden utilizar en niños lactantes, pre-escolares y escolares para evitar el retiro de los puntos que en este rango de edad causan ansiedad y dolor?

El calibre del hilo a utilizar y su permanencia varían de acuerdo a la zona anatómica ${ }^{2,3,10,11}$ (Ver Tabla 3); los puntos deben retirarse lo antes posible. Sin embargo, si se retiran demasiado pronto la herida se abre, y si se dejan demasiado tiempo el túnel que crea el hilo se epitelizará y dejará una marca permanente.

Tabla 2. Tipos de sutura de hilo

\begin{tabular}{|c|c|c|c|c|}
\hline \multirow{7}{*}{ Permanencia en tejido } & Sutura & Configuración & $\begin{array}{c}\text { Tiempo de } \\
\text { degradación }\end{array}$ & Indicaciones \\
\hline \multirow{4}{*}{ No absorbible } & Seda & Multifilamento & - & $\begin{array}{c}\text { Suturas cutáneas, ligaduras de } \\
\text { vasos }\end{array}$ \\
\cline { 2 - 5 } & Nylon & Monofilamento & - & $\begin{array}{c}\text { Suturas cutáneas precisas, sutura } \\
\text { tendinosa }\end{array}$ \\
\cline { 2 - 5 } & Polipropileno & Monofilamento & - & Sutura intradérmica, sutura \\
tendinosa
\end{tabular}

Fuente: autores 
Mayo - agosto

Tabla 3. Calibres de sutura y tiempo de retiro de puntos en piel

\begin{tabular}{|l|c|c|c|}
\hline Área suturada & $\begin{array}{c}\text { Calibre sutura } \\
\text { no absorbible }\end{array}$ & $\begin{array}{c}\text { Calibre } \\
\text { sutura } \\
\text { absorbible }\end{array}$ & $\begin{array}{c}\text { Tiempo de } \\
\text { retiro de } \\
\text { puntos(días) }\end{array}$ \\
\hline Párpados & 6.0 & - & $2-4$ \\
\hline Cara & $5.0 / 6.0$ & $4.0 / 5.0$ & $4-6$ \\
\hline Cuello & 4.0 & 4.0 & $5-7$ \\
\hline Tronco & $3.0 / 4.0$ & $3.0 / 4.0$ & $7-12$ \\
\hline Extremidades & $3.0 / 4.0$ & 4.0 & $10-14$ \\
\hline $\begin{array}{l}\text { Cuero } \\
\text { cabelludo }\end{array}$ & 3.0 & $3.0 / 4.0$ & $10-14$ \\
\hline Mano & 4.0 & $4.0 / 5.0$ & $7-10$ \\
\hline $\begin{array}{l}\text { Sobre } \\
\text { articulación }\end{array}$ & 3.0 & $3.0 / 4.0$ & $14-21$ \\
\hline
\end{tabular}

Fuente: autores

\section{Principios para un cierre óptimo de heridas}

Cuando se va a realizar una sutura, se deben conocer los diferentes tejidos que conforman los bordes de la herida (Ver Figura 6), de tal forma que se aproximen con sus mismas características y lograr una cicatrización correcta. Para lograr el objetivo de un óptimo cierre se cuenta con los siguientes principios básicos:

1. Cierre sin tensión: cuando existe tensión en el cierre de una herida, disminuye la vascularización de los bordes generando isquemia de la piel, limitando el proceso de cicatrización y aumentando la susceptibilidad a infecciones.

2. Eversión de los bordes de la herida: con el tiempo, por la contracción de la cicatriz, ésta tiende a aplanarse; si inicialmente se dejaron los bordes ligeramente elevados, los bordes se nivelarán con la piel indemne y el resultado estético será más aceptable.

3. Cierre por planos: es necesario solo cuando la herida es muy profunda y compromete otros tejidos además de la dermis e hipodermis, que de no ser afrontados generarán más tensión en los bordes de la piel y la formación de un espacio muerto donde se pueden crear hematomas o seromas.

4. Tipo de sutura: la elección adecuada del material de sutura y el grosor de hilo se realiza según el área corporal donde se encuentra la herida.
Principios en técnicas de suturas de piel: una guía para estudiantes

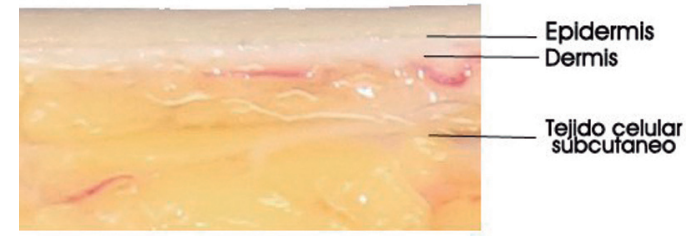

Figura 6. Composición macroscópica de la piel. Se muestra cada una de las capas de la piel (Epidermis y Dermis); además del tejido celular subcutáneo. Dependiente de la profundidad de la herida dependerá si se realiza el cierre por planos.

Fuente: autores

\section{Pasos para suturar una herida}

El paciente debe estar en una posición cómoda que exponga adecuadamente el área a suturar, teniendo una buena iluminación de la herida y los instrumentos necesarios. Se sigue un orden empezando por la asepsia hasta el cubrimiento de la herida suturada12,13:

1. Limpiar alrededor de la herida con un antiséptico local (clorexidina o yodopovidona).

2. Aplicación de anestésico local, atravesando la aguja por los bordes cruentos de la herida.

3. Lavado exhaustivo con SSN $0.9 \%$ o agua estéril aplicando presión para barrer detritos y cuerpos extraños.

4. Colocación de campos estériles.

5. Remodelación de los bordes de la piel necróticos o macerados.

6. Cierre de la herida con sutura de hilo según técnica de sutura elegida.

7. Realización del nudo con doble lazada inicialmente y luego lazadas simples asegurándose de formar nudos y no nudas.

8. Colocación de puntos necesarios hasta obtener un adecuado afrontamiento.

9. Cubrir herida suturada con apósito estéril.

Para la realización de una adecuada sutura se debe tener en cuenta los siguientes principios:

\section{Conformacion de nudo - lazada}

Para realizar una sutura de piel con hilo se deben diferenciar los conceptos de lazada y nudo, dado que el nudo ajusta la sutura y no permite que se suelte, mientras que la lazada no. 


\section{Lazada o seminudo}

Se forma un bucle con el segmento proximal del hilo y posteriormente, el segmento distal del hilo debe pasar por dentro del bucle; al traccionar los cabos del hilo en forma paralela a nivel del nudo, se debe formar una lazada plana que le dará estabilidad. Si se traccionan los hilos en direcciones opuestas y cruzándose al nivel del nudo, se formará una "media vuelta" que no es una lazada y puede aflojar el nudo o romper la sutura ${ }^{14}$ (Ver Figura 7 ).
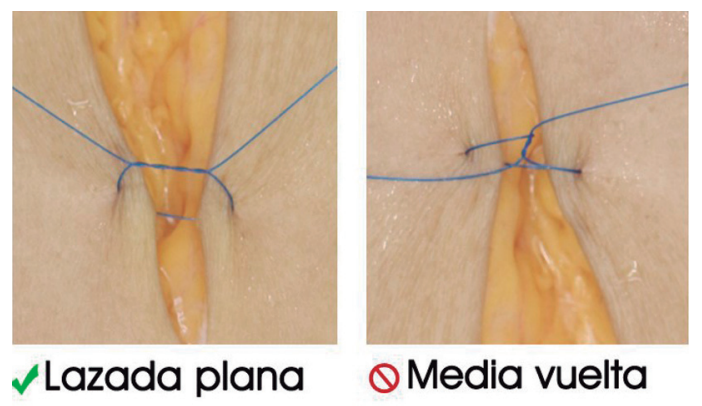

Figura 7. Lazada: pasar uno de los extremos del hilo por dentro de un bucle formado con el otro extremo del hilo y posteriormente traccionar los dos cabos del hilo. Media vuelta: esta se formara si al pasar uno de los extremos del hilo por dentro del bucle conformado por el otro extremo del hilo se traccionan en dirección incorrecta. Fuente: autores

\section{Nudo}

Un nudo se compone por lo menos de dos lazadas, donde la primera lazada es plana, a partir de un doble bucle y las siguientes lazadas son sencillas con un solo bucle. Los nudos se pueden hacer con las manos o con un porta agujas. Esta doble lazada inicial ayuda a asegurar la sutura mientras se realiza la siguiente lazada sencilla y se ajusta el nudo. Se realizan dos, tres o cuatro lazadas, dependiendo del tipo de sutura y de la tensión de la herida ${ }^{14}$ (Ver Figura 8). El nudo se debe asegurar lo suficiente para crear eversión de los bordes libres de la herida, teniendo precaución de no ajustar demasiado, dado que puede producir isquemia de la piel.

Para un afrontamiento simétrico de una herida lineal, se utiliza la técnica de cierre por mitades, donde se coloca un primer punto sencillo en el centro de la herida y luego se colocan puntos sencillos en las mitades restantes, logrando un cierre paulatino y uniforme de todos los bordes de la piel (Ver Figura 9).

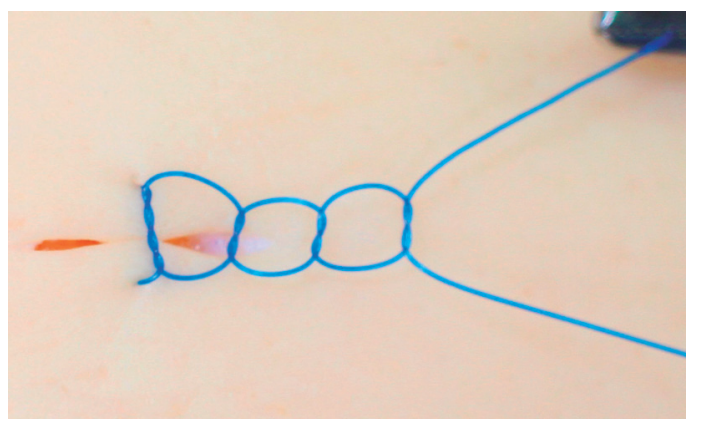

Figura 8. Realización de nudo. Formación de varios nudos donde la primera lazada es plana a partir de un doble bucle y las siguientes son con un solo bucle.

Fuente: autores

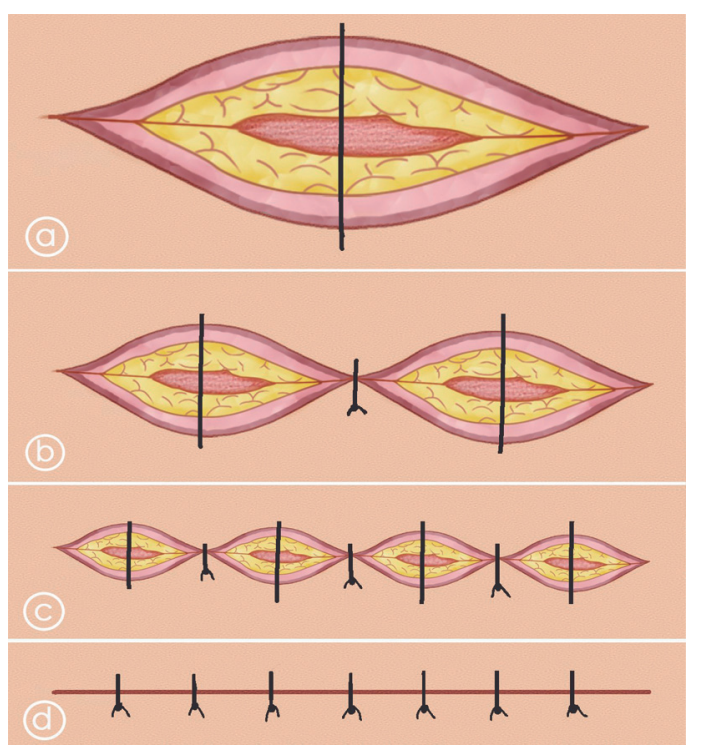

Figura 9. Cierre por mitades de una herida. a. Primer punto en la mitad de la toda la longitud de la herida. b. Segundo punto en la mitad resultante a cada lado del primer punto. c. Colocación de puntos sucesivamente por mitades. d. Hasta completar el cierre de la herida.

Fuente: autores

\section{Puntos básicos para cierre de heridas en piel}

Las técnicas de sutura básicas de piel con sutura de hilo, consisten en la realización de puntos separados o continuos, que tendrán una indicación especifica según el tipo de herida y la región corporal que se esté interviniendo.

\section{Puntos subdérmicos}

Los bordes de una herida que son alineados sin tensión epitelizan con menos formación de cicatriz, 
ésto se logra con la colocación de puntos subdérmicos que liberan tensión en la dermis y epidermis, para luego colocar los puntos en piel. Los cuales se hacen de forma convencional o invertidos. Los puntos invertidos se inician ingresando la aguja por uno de los bordes cruentos a nivel subcutáneo profundo y se re direcciona siguiendo el arco de ésta a través de la dermis, para salir a nivel de la porción superior de la dermis, para luego hacer el mismo movimiento en el borde cruento opuesto ingresando superior y saliendo en la parte cruenta profunda, de tal forma que al atar el nudo, éste va a quedar en la porción profunda de la herida con pocas posibilidades de extrusión. En el punto subdérmico convencional, el nudo queda en la parte superior. Se cortan los cabos de sutura cortos para disminuir la reacción a cuerpo extraño3 (Ver Figura 10).
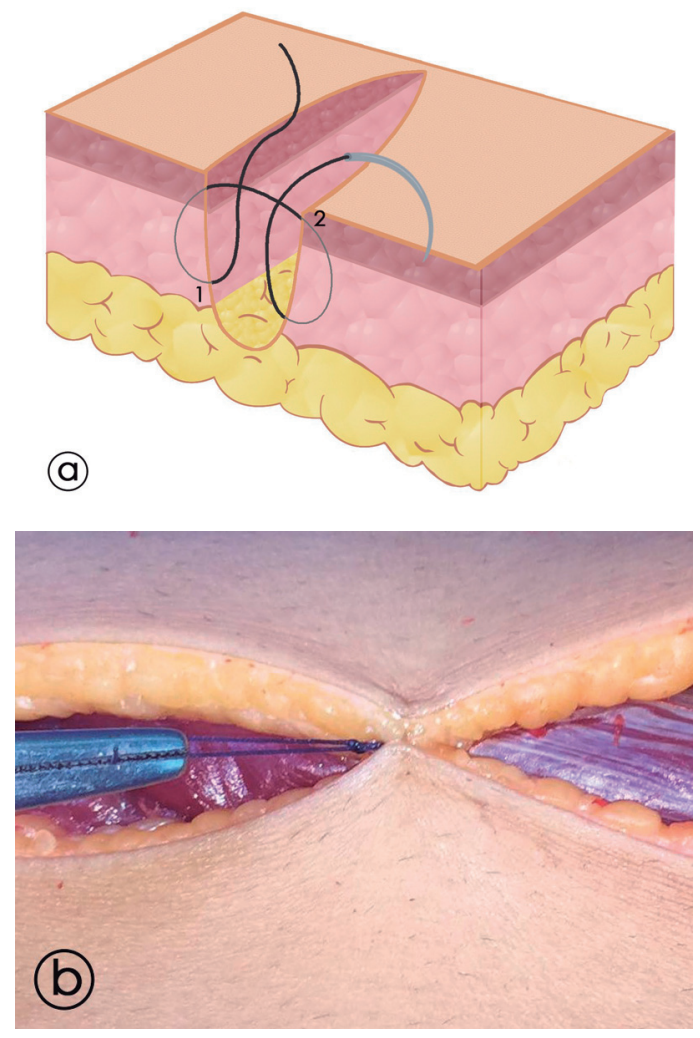

Figura 10. Punto subdérmico invertido. a) 1. Introducción de aguja de profundo a superior, 2. En borde opuesto paso de aguja de superior a inferior. b) Conformación de Punto subdérmico invertido con afrontamiento de bordes.

Fuente: autores

\section{Puntos simples o separados}

Son los puntos más usados en cirugía. Para realizarlos se coloca la punta de la aguja $90^{\circ}$ perpendicular a la superficie de la piel (Ver Figura 11) y 3 a $5 \mathrm{~mm}$ del borde de la herida; posteriormente se dirige la aguja desde el exterior hacia el interior pasado por dermis e hipodermis, y se gira a través del tejido hacia el lado opuesto del borde de la herida, siguiendo el arco de movimiento de la aguja y con un movimiento suave de la muñeca. En el lado opuesto del borde se realiza la misma operación pero desde el interior hacia el exterior y se saca la aguja la misma distancia donde se ingresó en el borde inicial, atando con un nudo. Este paso se puede realizar en dos tiempos teniendo precaución de ingresar en el lado opuesto al mismo nivel de donde previamente se pasó la aguja, de lo contrario, se formará una irregularidad en los bordes de la herida al momento del cierre, creando un escalón. La distancia entre los puntos debe ser equidistante ${ }^{3}$ (Ver Figura 12).

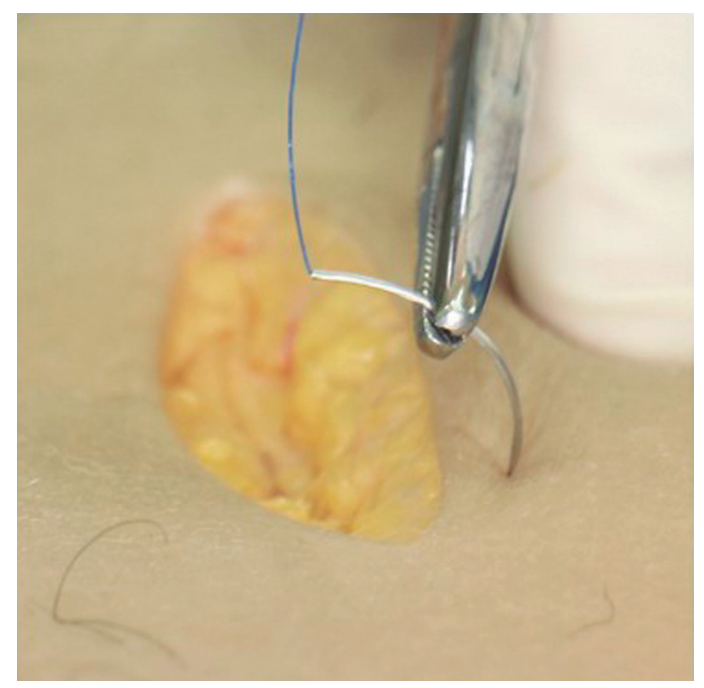

Figura 11. Posición para la correcta entrada de la aguja en la piel. Se coloca la punta de la aguja $90^{\circ}$ perpendicular a la superficie de la piel y de 3 a $5 \mathrm{~mm}$ del borde de la herida.

Fuente: autores
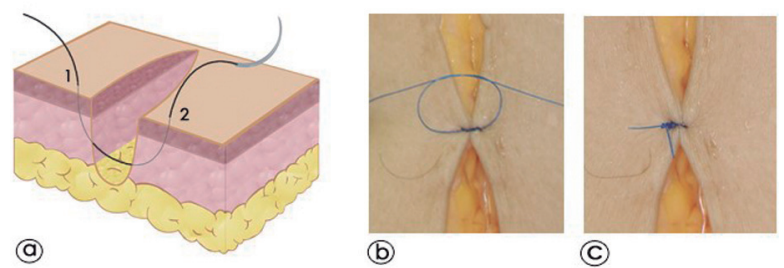

Figura 12. Punto simple. a) Esquema pasó punto simple. b) Paso del primer punto con lazada plana y luego conformación de nudo. c) Punto fijo dejando a un lado el nudo de la herida.

Fuente: autores 


\section{Punto colchonero vertical o de blair donati}

Utilizado para disminuir la tensión en la herida y evertir los bordes. Para la realización de este punto se emplea la nemotecnia "Lejos-Lejos, CercaCerca" (Ver Figura 13) iniciando con introducción de la aguja en la epidermis 5 a $10 \mathrm{~mm}$ del borde de la herida (Lejos) y se dirige hacia abajo tomando una parte generosa de la dermis, se retoma con el porta agujas en el borde vertical libre de la herida para luego pasarla en el borde opuesto tomando la porción de la dermis y direccionando la aguja hacia la superficie, saliendo a la misma distancia inicial en posición "Lejos" del borde de la herida. Posteriormente se cambia la dirección de la aguja en el porta agujas y se penetra en el mismo borde donde se acaba de obtener, pero a una distancia "Cerca" del borde de la herida, alrededor de 2 a $3 \mathrm{~mm}$, tomando una porción más superficial de la dermis; se avanza la aguja al borde opuesto y se saca a la superficie a la misma distancia "Cerca" que se dejó en el borde opuesto para finalmente atar el nudo (Ver Figura 14). Para mejores resultados, todos los puntos de entrada y de salida deben ser equidistantes y seguir una línea recta perpendicular a los bordes de la herida ${ }^{16}$.

\section{Punto colchonero horizontal}

Utilizado para disminuir y redistribuir tensión, cerrar el tercer espacio y para evertir los bordes de la herida. Inicialmente se coloca la aguja 4-10 mm del borde de la herida, pasándola por la epidermis y dermis hacia el lado contralateral, saliendo en ese lado a una distancia del borde de la herida igual a la tomada en el lado inicial. En ese mismo lado, se reingresa a la epidermis $3-5 \mathrm{~mm}$ lateral a la salida del último punto y a la misma distancia manejada hacia el borde de la herida; se dirige la aguja por la dermis hacia el lado opuesto, saliendo por al exterior lateral al primer punto de ingreso en la piel y se ajusta con nudos (Ver Figura 15). Cuando se completa, la sutura expuesta en la piel se dispone paralela al borde de la herida17 (Ver Figura 16).

Hay un mayor riesgo de isquemia del plexo subdérmico con este tipo de puntos comparado con los puntos simples y los colchoneros verticales, por lo que no se deben usar en tejidos pobremente vascularizados ${ }^{17}$.

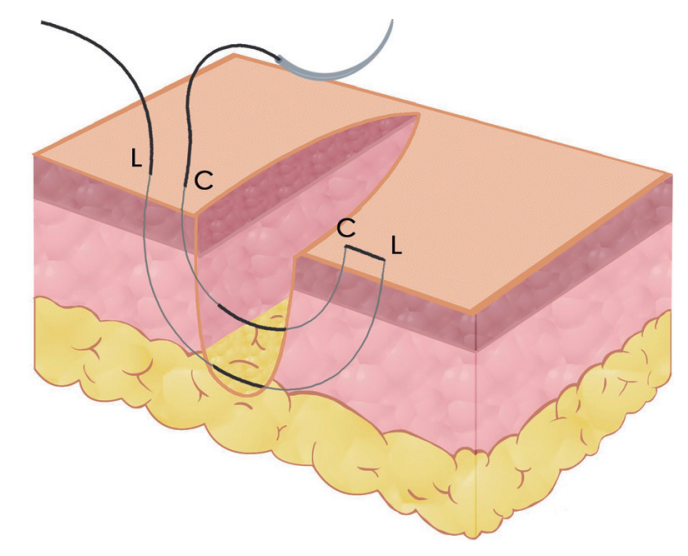

Figura 13. Punto de colchonero vertical. Esquema de nemotecnia para su realización (Lejos-Lejos, Cerca-Cerca).

Fuente: autores

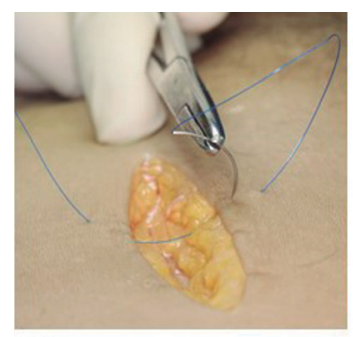

(a)

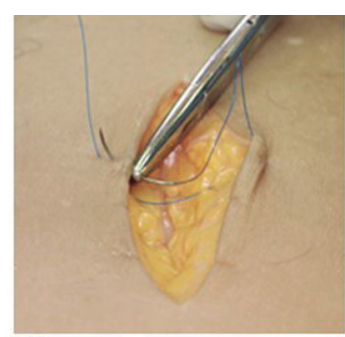

(b)

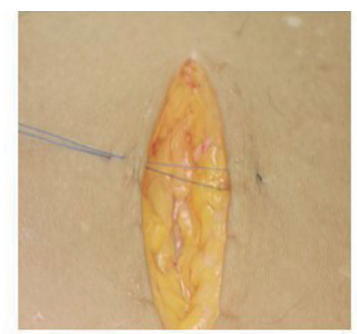

(C)

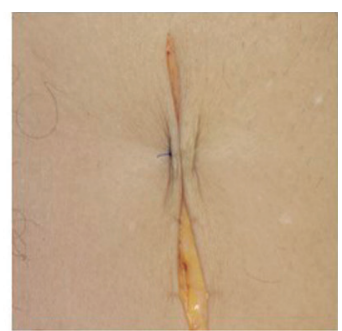

(d)

Figura 14. Punto colchonero vertical. a) Aso de punto Lejos -Lejos. b) Paso de punto Cerca - Cerca. c y d) Ajuste y nudo con eversión de bordes.

Fuente: autores 


\section{Punto de colgajo o colchonero horizontal semienterrado}

Los puntos de colchonero horizontal se pueden colocar con la mitad de la sutura dentro de la dermis, evitando la isquemia en los bordes de la herida y logrando a su vez eversión de los mismos. Se utilizan habitualmente cuando hay heridas con bordes angulados, dejando la parte del punto subdérmico en el borde de la punta del colgajo traumático o borde angulado y saliendo al exterior en el borde opuesto, donde encajará el vértice con la punta del borde de la herida (Ver Figura 17). La primer entrada es igual que un colchonero horizontal, pero en el lado opuesto, se pasa la aguja de forma paralela a la superficie de la epidermis, entrando y saliendo por los bordes de la dermis. Finalmente, se saca la aguja en el borde opuesto lateral a la primera entrada ${ }^{11,15}$ (Ver Figura 18).

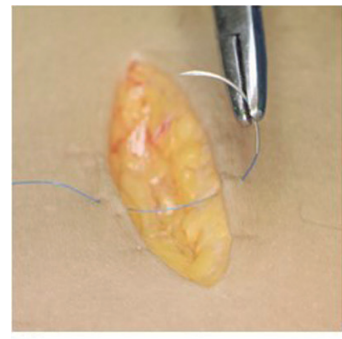

(a)

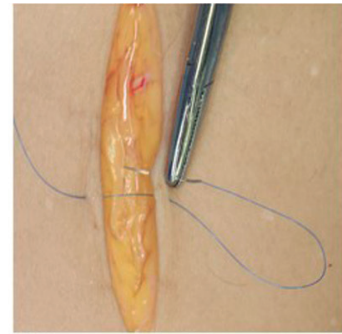

(b)

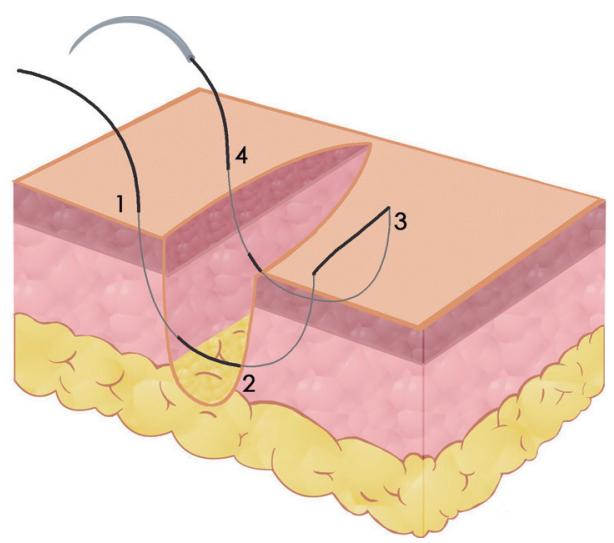

Figura 15. Esquema como se debe realizar el punto colchonero horizontal.

Fuente: autores

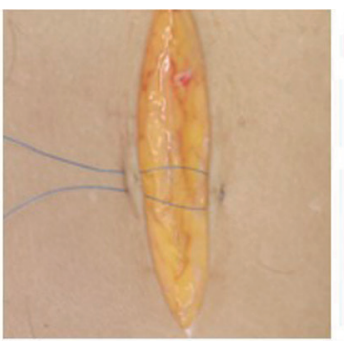

(c)

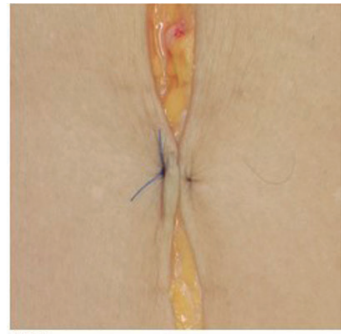

(d)

Figura 16. Punto colchonero horizontal. a) Paso primer punto. b) Reingreso de aguja lateral a salida previa. $\mathrm{c}$ y d) Ajuste y nudo. Fuente: autores

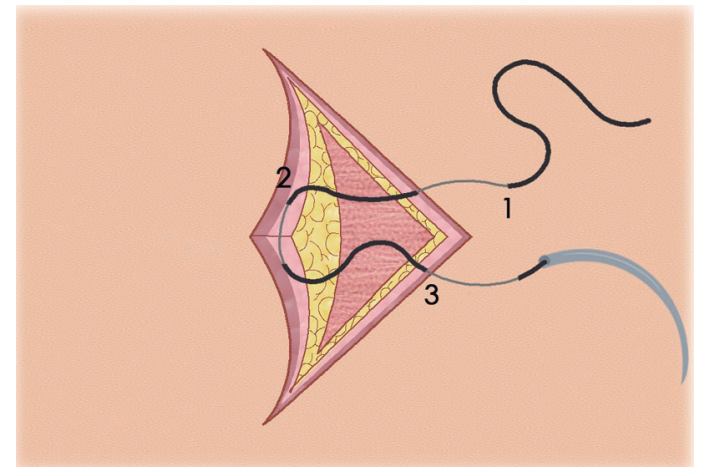

Figura 17. Esquema para la realización de punto de colgajo. 1. Entrada de la aguja. 2. Punto paralelo a la epidermis pero atravesando la dermis. 3. Punto de salida de la aguja. Fuente: autores

\section{Sutura continua intradérmica o subcuticular}

Son puntos simples intradérmicos continuos sin nudos, lo que hace que sea una técnica de sutura más rápida pero no tan fuerte como los puntos separados, por lo que debe utilizarse cuando esté indicada. No se emplea como única forma de cierre en heridas con tensión moderada a significativa, porque los puntos continuos pueden causar isquemia del plexo subdérmico superficial y lesionar los tejidos. Además, en caso de formación de hematoma que requiera drenaje, va a ser necesario retirar toda la sutura, que de haber sido con puntos separados, solo se retirarán los puntos necesarios para el drenaje ${ }^{18}$.

Con esta técnica se obtiene un mejor resultado estético, dado que no hay salidas de los puntos a la superficie, permitiendo dejarlos por más tiempo y lograr un mejor proceso de cicatrización.

Se inicia colocando la aguja aproximadamente un $\mathrm{cm}$ distal de uno de los bordes finales de la herida, pasando la aguja para salir justo en el vértice de la herida, la cola de la sutura se repara con una mosquito para al final anudar (Ver Figura 19); posteriormente se direcciona la aguja de manera paralela a la epidermis y se pasa en uno de los bordes en la línea de unión de la epidermis con la dermis, saliendo $5 \mathrm{~mm}$ a $6 \mathrm{~mm}$ distal en el borde de la herida. Luego, se introduce la aguja en el lado opuesto en el mismo plano y con la misma disposición horizontal, a la misma altura del punto anterior, para lo cual se traspone 
la sutura al lado contrario como guía para ubicar el nivel del siguiente punto (Ver Figura 20). Siguiendo estos puntos de manera sucesiva, la sutura toma la configuración de un zigzag intradérmico que afronta muy bien los bordes de la herida y finalmente se sale distal al borde final de la herida y se ata la sutura sobre sí misma, formando un bucle con una doble lazada y lazadas simples posteriores para asegurar el nudo?. En suturas intradérmicas largas se pueden realizar puentes de sutura (salida de la sutura desde dermis a piel y nuevamente ingreso desde piel a dermis desde el lado contralar) para facilitar su retiro.

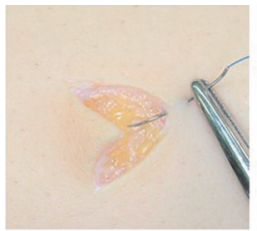

(a)

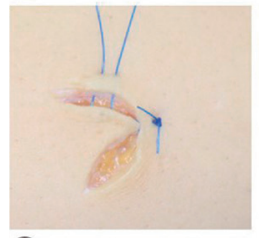

(d)

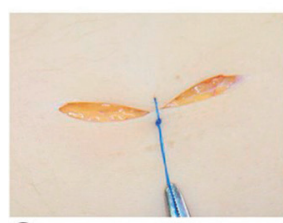

(a)

Figura 18. Punto de colgajo. a) Paso primer punto en borde opuesto a herida angulada. b) Paso de aguja por vértice de herida angulada. c y d) Ajuste y nudo. e. y f) Colocación de puntos simples necesarios para afrontamiento de todos los bordes.

Fuente: autores.

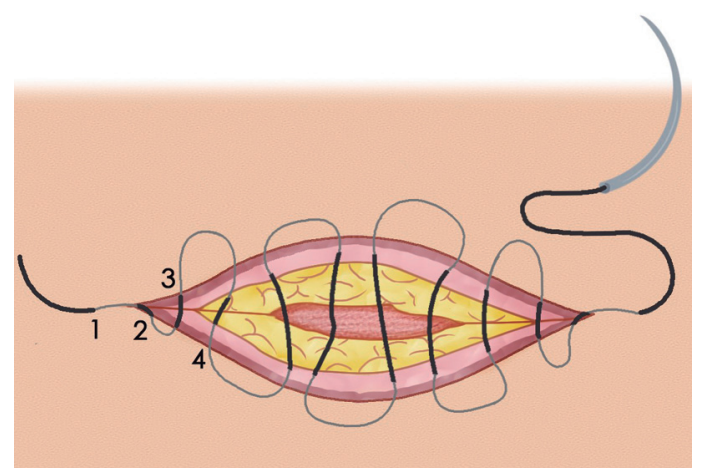

Figura 19. Esquema conformación sutura intradérmica. 1. Entrada de la aguja a nivel del vértice 2. Punto cercano al vértice 3 . Punto del lado contrario del punto cercano al vértice. 4 . Continuación de la sutura en un plano intradérmico en forma de zigzag.

Fuente: autores.

\section{Retiro de puntos}

Las suturas deben ser retiradas en un tiempo suficiente que permita el desarrollo inicial de la cicatrización y la fuerza de tensión en la herida; si se dejan por mucho tiempo, los puntos crean marcas en la piel, predisponen a infección de tejidos blandos y ralentizan el proceso de cicatrización ${ }^{12,14}$ (Ver Tabla 4). Para retirar un punto, se tracciona la cola de la punta de la sutura con unas pinzas y se corta uno de los lados de la sutura por debajo del nudo, luego se hala el hilo hacia arriba y se retira en su totalidad.

Tabla 4. Tiempo para retiro de puntos según área corporal

\begin{tabular}{|l|c|}
\hline \multicolumn{1}{|c|}{ Área suturada } & $\begin{array}{c}\text { Tiempo de retiro de } \\
\text { puntos (días) }\end{array}$ \\
\hline Párpados & $2-4$ \\
\hline Cara & $4-6$ \\
\hline Cuello & $5-7$ \\
\hline Tronco & $7-12$ \\
\hline Extremidades & $10-14$ \\
\hline Cuero cabelludo & $10-14$ \\
\hline Mano & $7-10$ \\
\hline Sobre articulación & $14-21$ \\
\hline
\end{tabular}

Fuente: autores

\section{Cuidados de la herida}

Si es una herida en un área de alta tensión, se puede reforzar con cintas adhesivas que ayudarán a aproximar los bordes ${ }^{19}$. Se cubre la herida con un apósito estéril de gasa, si se sospecha de posible salida de exudado, o se puede dejar cubierta solo con cintas adhesivas.

Instrucciones al paciente y los familiares respecto a los cuidados de la herida ${ }^{20}$ :

- No mojar ni quitar el vendaje por 24 a 48 horas, manteniendo la herida limpia y seca; solo se cambiará el apósito si se ensucia o moja con exudado de la herida.

- Primera curación 24 a 48 horas para comprobar si hay signos de infección.

- Informar de la posibilidad de infección (calor, rubor, dolor, mal olor, fiebre, pus o secreción) y signos clínicos que se deben vigilar, que de estar presentes; el paciente debe asistir por urgencias.

- Lavar la herida diariamente con agua y jabón suave. 
- Proteger la herida con un apósito de cambio diario.

- Retirar los puntos de sutura en los días indicados.

- En caso de heridas con tiras adhesivas, no mojar la zona y retirarlas en un control a los cinco días.

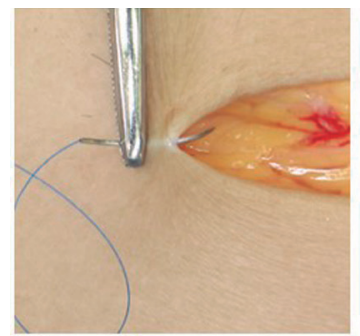

(a)

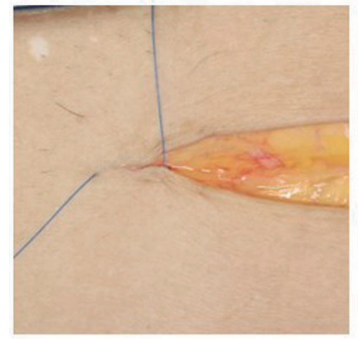

(e)

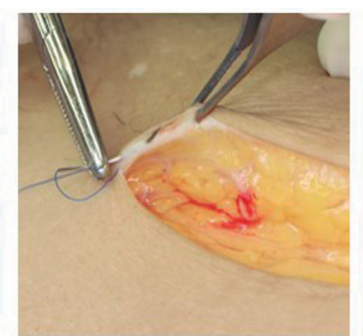

(b)

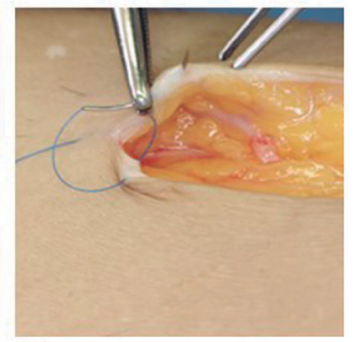

(f)
- Protección solar por seis a doce meses, en caso de exposición utilizar protector solar dos veces al día.

- Adyuvancia con cremas cicatrizantes que estimulan la cicatrización y mejoran el proceso de epitelización.

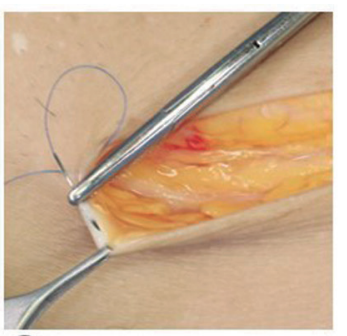

(c)

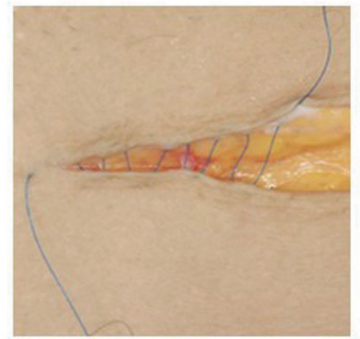

(9)

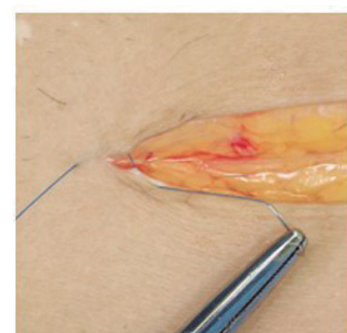

(d)

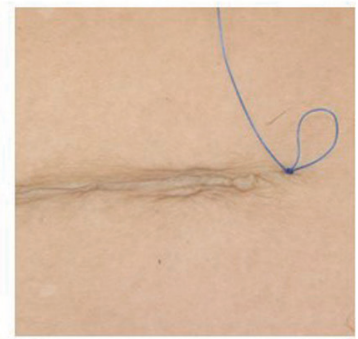

(h)

Figura 20. Sutura intradérmica. a) Ingreso de aguja en vértice de la herida. b) Primer punto cercano al vértice. c) Punto en borde opuesto cercano al vértice. d) Tracción y ajuste de sutura. e) Proyectar siguiente punto colocando sutura en borde opuesto. f y g) Colocación de puntos siguientes. h) Cierre completo de herida y ajuste con nudo.

Fuente: autores

\section{Indicación de manejo por especialista}

Requieren manejo por especialista aquellas heridas complejas, irregulares con avulsión de tejidos blandos y compromiso de área especial como párpados o labios. También se deben referir aquellas heridas altamente contaminadas y con gran tejido desvitalizado que requieran un minucioso desbridamiento quirúrgico.

\section{Conclusiones}

Los resultados en la realización de una sutura de piel dependerán de un adecuado conocimiento y uso del material e instrumental quirúrgico, además del dominio de los principios básicos de la técnica quirúrgica, teniendo en cuenta la localización y complejidad de la herida para definir el cierre por planos, y el tipo de punto con su adecuada realización del nudo, para lograr un cierre sin tensión y eversión de los bordes de la herida. Asimismo, se debe incluir el uso de anestésicos locales y los cuidados posquirúrgicos para lograr buenos resultados.

\section{Conflictos de interés}

Los autores declaran no tener conflictos de interés de ningún tipo.

\section{Referencias bibliográficas}

1. Thorne, C. 2007. Techniques and principles in plastic surgery. En: Wolters Kluwer. Grabb and Smith's plastic surgery. Philadelphia: Wolters Kluwer; 2013. p. 3-14.

2. Goldman G, Travelute C. 2012. Wound closure materials and instruments. En: Elsevier. Dermatology. 3ed. Edimburgo: Elsevier; 2012. p. 2353 - 63.

3. Usatine R, Coates W. Laceration and incisión repair. En: Mosby. Pfenninger and fowler's procedures for primary care. 3ed. Philadelphia: Mosby; 2011. p. 157 - 169.

4. Ollague J, Zabielinski M, Nouri K. Anesthesia in dermatologic surgery. En: Blackwell. Dermatologic surgery, step by step. Oxford: Blackwell; 2013. p. $20-25$.

5. McGee D. Local and topical anesthesia. En: Saunders. Roberts and hedges' clinical procedures in emergency medicine. Philadelphia: Saunders; 2014. p. 519-540.

6. Vinycomb T, Sahhar L. Comparison of local anesthetics for digital nerve blocks: a systematic review. J Hand Surg Am. 2014;39(4): 744-751.

7. Chowdhry S, Seidenstricker L, Cooney DS, Hazani R, Wilhelmi B. Do not use epinephrine in digital blocks: myth or truth? Part II: A retrospective review of 1111 cases. Plast Reconstr Surg. 2010;126(6): 2031-4. 


\section{González-Cely A, Miranda-Díaz A, Alviar J}

8. Simon B, Hern G. Wound management principles. En: Marx J, Hockbergr R, Walls R. Rosen's Emergency medicine. 8va ed. Philadelphia: Saunders; 2014 p: 751- 766.

9. Hochberg J, Meyer K, Marion M. Suture choice and other methods of skin closure. Surg Clin North Am. 2009; 89(3):627-41.

10. Choudhary S, Nouri K, Jungcharoensukying P, Elsaie M. Suturing techniques. En: Dermatologic Surgery, Step by Step. Nouri K. Oxford: Blackwell; 2012. p: 25-9.

11. Kandel E, Bennett R. The effect of stitch type on flap tip blood flow. J Am Acad Dermatol. 2001;44(2):265-72.

12. Richard L, Smith Z. Methods of wound closure. En: Roberts J. Roberts and Hedges' Clinical Procedures in Emergency Medicine. 6ta ed. Philadelphia: Saunders; 2014 p: 644-89.

13. Richard L, Smith Z. Principles of wound management. En: Roberts J. Roberts and Hedges' Clinical Procedures in Emergency Medicine. 6ta ed. Philadelphia: Saunders; 2014. p: 611-43.

14. Hernandez C, Jimenez R, Busto M, Zabaleta J, Aguinagalde

\section{MÉD.UIS. 2018;31(2):65-76}

B, Zulaika N, et al. Manual sobre suturas, ligaduras, nudos y drenajes. Donostia - San Sebastián: Osakidetza; 2007.

15. Wirth C, Bouletreau P. Cirugía de los traumatismos de los tejidos blandos y de las heridas faciales. EMC - Cirugía plástica reparadora y estética. 2012;20(1):1-14.

16. Revol M., Servant J.-M. Suture. EMC - Techniques chirurgicales - Chirurgie plastique reconstructrice et esthétique. 2010;5(1):18.

17. Moy R, Waldman B, Hein D. A review of sutures and suturing techniques. J Dermatol Surg Oncol. 1992;18(9):785-95.

18. Hochberg J, Murray G. Principles of operative surgery. In: Townsend C, Beauchamp D, editors. Sabiston Textbook of surgery. Texas: Elsevier Saunders; 2012. p. 253-63.

19. Efron G, Ger R. Use of adhesive tape (Steri-Strips) to secure skin grafts. Am J Surg. 1968; 116(3):474.

20. Blanco A, Fortet C, Pata R, Olaso S, Guztke M. Suturas básicas y avanzadas en cirugía menor. Semergen. 2002;28(1):89-100. 